\title{
Functional hemispheric differences for the categorization of global and local information in naturalistic stimuli
}

\author{
Ronald Hübner ${ }^{\circ}$, Tobias Studer
}

\begin{abstract}
A B S T R ACT
Up to now functional hemispheric asymmetries for global/local processing have mainly been investigated with hierarchical letters as stimuli. In the present study, three experiments were conducted to examine whether corresponding visual-field (VF) effects can also be obtained with more naturalistic stimuli. To this end, images of animals with a pattern placed on their body were displayed as stimuli. The task for the global level and for the local level was to categorize the animals and the patterns, respectively. As a result, VF-effects were also found for these stimuli and tasks, It is concluded that the hemispheric differences observed for hierarchical letters also hold for naturalistic stimuli.
\end{abstract}

\section{Introduction}

For more than twenty years, it has been proposed that the cerebral hemispheres differ with respect to their capacities for analytic and holistic processing. Mainly based on neuropsychological studies with split-brain patients, it has been hypothesized that the left hemisphere processes information more analytically. whereas the right hemisphere processes stimuli more holistically (for an early overview see Bradshaw \& Nettleton, 1981). The investigation of this hypothesis has been considerably intensified after Navon's (1977) introduction of hierarchical letters as stimuli, i.e. of global letters constructed from local letters. Although Navon himself was mainly interested in the time course of global and local processing, other researchers soon realized that hierarchical letters might also be ideal for investigating hemispheric differences with respect to analytic and holistic processing. One merely has to assume that these modes correspond to the processing of local and global letters, respectively (for a critical view in this respect see Kimchi, 1992). Accordingly, in behavioral studies one could present a hierarchical letter in the left visual field (LVF) or in the right visual field (RVF), and require the participants to categorize the local or the global letter. If the proposed hemispheric differences really exist, then the categorization of local letters should be faster for stimuli presented in the RVF than for those appearing in the LVF, whereas the opposite should hold for global letters. Indeed, only two years after Navon's (1977) paper, Martin (1979) reported such a study in which she found the expected visual-field (VF) effects.

•Universität Konstanz, Fachbereich Psychologie, D-78457 Konstanz

E-Mail: Ronald.Huebner@uni-konstanz.de
Meanwhile, hierarchical letters have successfully been applied in numerous behavioral studies (for overviews see Hübner \& Volberg, 2005; Van Kleeck, 1989; Yovel, Yovel, \& Levy, 2001), electrophysiological studies (e.g. Han, Fan, Chen, \& Zhuo, 1999; Heinze \& Münte, 1993; Malinowski, Hübner, Keil, \& Gruber, 2002; Volberg \& Hübner, 2004), neuropsychological studies (e.g. Delis, Robertson, \& Efron, 1986; Lux, Thimm, Marshall, \& Fink, 2006; Robertson \& Lamb, 1991), and brain imaging studies (e.g. Fink et al., 1996. 1997; Heinze, Hinrichs, Scholz, Burchert, \& Mangun, 1998; Lux et al., 2004). However, hierarchical letters ${ }^{1}$ are rather abstract and artificial (cf. Pomerantz, 1981). Therefore, although there have been some attempts to generalize the VF-effect hypothesis to the auditory domain (e.g. List \& Justus. 2007), what is still missing is evidence that the results obtained with hierarchical letters can be generalized to naturalistic objects.

In most global/local studies hierarchical letter stimuli were preferred, because they have some useful properties. First of all, they can easily be constructed. Moreover, the content at one level can be varied independently from that at the other level. This orthogonality property is crucial for preventing contingencies, i.e. the priming of global forms by local forms, and vice versa. Finally, global and local forms are not only independent, they can also be exchanged. That is, certain content can occur at one level, at the other, or even at both levels. This aspect is important if one wants to investigate which of the two levels of a hierarchical stimulus is processed fas-

\footnotetext{
Although hierarchical letters were used in the majority of studies, it should be noted that hierarchical stimuli with abstract and symbolic forms other than letters have also been applied in some studlies (e.g. simple geometric shapes). For simplicity. though, we will mostly use the term 'hierarchical letters' even when we mean the whole class of such artificial stimuli.
} 
ter, because without this property level and respective content are confounded.

However, the objects we usually encounter in our environment are different from hierarchical letters. Thus, the challenge is to find objects that are natural but nevertheless retain some of the useful properties of hierarchical letters. First of all, it should be noted that for the investigation of hemispheric asymmetries exchangeability is not mandatory, because one is only interested in response time differences between the hemispheres within one level and not in response time differences between levels. VF-effects can therefore be examined with stimuli that have a specific content at each level. One would merely have to demonstrate that the specific content at each level does not produce VF-effects without being part of the hierarchical structure. Orthogonality, however, would be a desirable property, because, as already mentioned, information at one level should not be correlated with the information at the other level. Therefore, we were looking for objects that appear quite natural but retain at the same time the orthogonality property.

As a first step, we considered stimuli usually applied in the field of object recognition and object categorization. Also in these areas global/local processing has been investigated to some extent. For instance, it has been shown that for the identification of living objects global information is more important than local information. whereas the opposite holds for the identification of non-living objects (e.g. Lag, Hveem, Ruud, \& Laeng, 2006; Thomas \& Forde, 2006). The stimuli applied in such studies were mostly images of naturalistic objects. Therefore, we decided to use such stimuli also for our experiments. However, the question was which of their features correspond to the global stimulus level and which to the local one.

With respect to the global level, it seems reasonable to assume that it corresponds to the overall object shape. Behavioral (e.g. Arguin \& Saumier, 2004; Johnston \& Hayes, 2000; Laeng, Shah, \& Kosslyn, 1999: Large \& McMullen, 2006) as well as neuropsychological (e.g. Behrmann, Peterson, Moscovitch, \& Suzuki. 2006; Humphreys et al., 1994) evidence suggests that the overall shape of familiar objects is perceived holistically, i.e. that the overall shape is coded and represented independently of its parts. This view is also supported by results showing that the global shape of an object is already represented mentally at an early stage of processing (cf. Humphreys, 1998; Tyler et al., 2004).

In view of the objects applied in these studies, we constructed shaded images of animals as stimuli and assumed that, because these stimuli represent familiar naturalistic objects, their overall shape is encoded holistically. Accordingly, the task for the global level was to categorize the animals.

After we had decided to regard the overall shape of the animals as the global level, the question was what should be used as the local level. One possibility would have been to consider the different body parts as the local level. However, this was inappropriate. Apart from the difficulty of finding a task for the object parts, the levels would not have been independent, because many parts are informative with respect to the object category, i.e. the global level. Facing these problems, we decided to place a pattern of certain elements on the body of the animals (see Fig. 1) and to regard the elements as the local level. Accordingly, the task for the local level was to categorize the elements. Most importantly, because all animals could be combined with each element type, it was possible to vary the contents of the two stimulus levels independently.

\section{Experiment 1}

In our first experiment, the stimuli were images of a cat, a dog. or a dolphin with a pattern of dots, lines, or ovals placed on their body (see Fig. 1). The global task was to categorize the displayed animal as dog or cat, and the local task was to categorize the pattern elements as dots or lines. Dolphins and ovals served as neutral components for the global and local task, respectively. Animals and elements were mapped to the response buttons in such a way that the stimuli were congruent, incongruent, or neutral. A stimulus was congruent or incongruent, when the animal and pattern were mapped to the same response or to different responses, respectively. A stimulus was neutral, when the content of the irrelevant level was not mapped to any response.

Given these stimuli, we expected similar VF-effects as those observed with hierarchical letters. That is, there should be a LVFadvantage for the animal categorization task, and a RVF-advantage for the pattern element categorization task.

\subsection{Method}

\subsubsection{Participants}

Sixteen persons ( 10 female; mean age 23.6 years) participated in the experiment. All were right-handed (by self report), and had normal or corrected-to-normal vision.

\subsubsection{Apparatus}

The stimuli were presented on a 21 "-color-monitor (Sony) with a resolution of $1024 \times 768$ pixels and a refresh rate of $85 \mathrm{~Hz}$. A personal computer (PC) served for controlling stimulus presentation and response registration.

\subsubsection{Stimuli}

Shaded images of cats, dogs, and dolphins served as stimuli. Their color was brown. The animals were also covered with a pattern of dot, line, or oval elements. Cats and dogs were combined with all three element types, whereas dolphins occurred only with dots or lines. Each animal/element combination was rendered in orientations of $60^{\circ}$ and $120^{\circ}$ in the depth plane. Then mirror images from each of these pictures were made. Thus, altogether. there were 32 different stimuli (12 dogs, 12 cats, 8 dolphins). Examples can be seen in Fig. 1. The stimuli were presented on a black background. The size of the stimuli depended on the animal and its orientation, respectively. Their height varied from $3.89^{\circ}$ to $4.62^{\circ}$, whereas, their width varied from $4.69^{\circ}$ to $5.58^{\circ}$. The stimuli were presented at an eccentricity of $2.79^{\circ}$ (center of the screen to center of the stimulus).

\subsubsection{Procedure}

The task for the global level was to categorize the animals as cats or dogs by pressing one out of two response buttons with the index or middle finger, respectively, of the same hand. Using the same buttons and fingers, the task for the local level was to categorize the pattern elements as dots or lines. Mapping of animals and pattern elements to response keys (or fingers), and responding hand were counterbalanced across participants. For instance, some of the participants had to respond to cat and dots with their index finger and to dog and lines with their middle finger of their right hand. Other participants responded with their left hand and/or had a different category-to-finger mapping. All four possible mappings were realized.

Each trial started with the appearance of a letter as cue for $300 \mathrm{~ms}$ at the center of the screen. A " $\mathrm{T}$ " (first letter of the German word 'Tier', which means animal) or " $M$ " (first letter of the German word 'Muster', which means pattern) indicated animal task (global) or pattern task (local), respectively. After a blank screen of $500 \mathrm{~ms}$ the stimulus was presented for $93 \mathrm{~ms}$ either to the left visual field (LVF), or to the right visual field (RVF) at an eccentricity of $2.79^{\circ}$ (center of the screen to center of the stimulus). $1000 \mathrm{~ms}$ after the response the next cue appeared. Errors were signaled by a tone. 


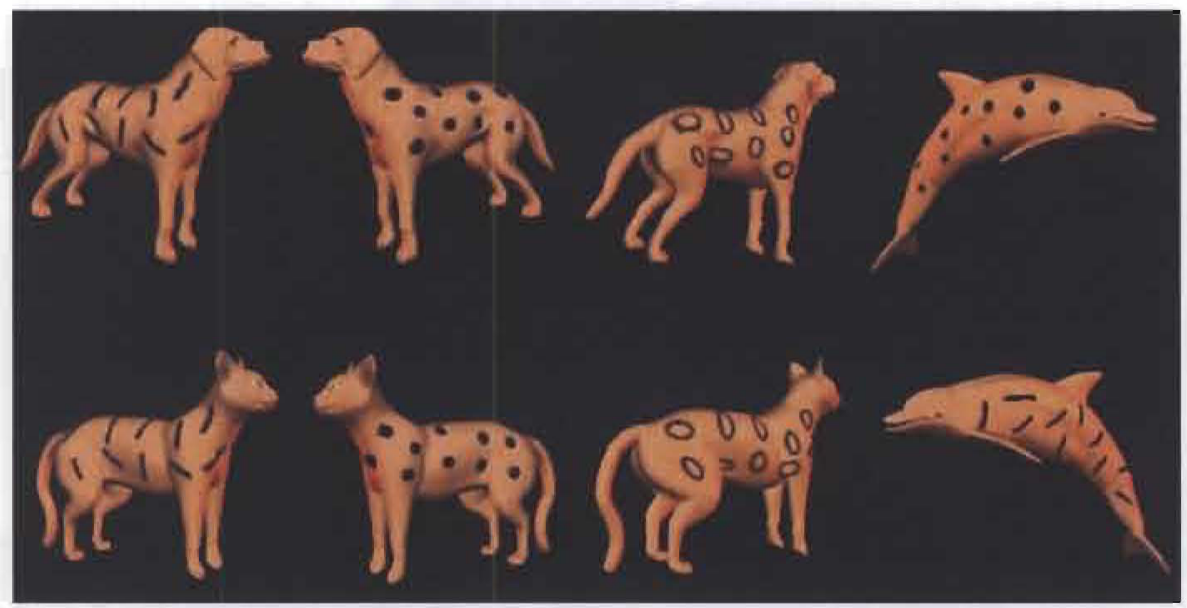

Fig. 1. Examples of stimuli used in Experiment 1. For further details see the text-

There were three factors: Visual field (LVF or RVF), target level (global or local), and congruency (congruent, neutral, or incongruent). All factors were randomized within each block of trials. Altogether there were 12 conditions. After one practice block, 10 blocks of 96 trials were run. Altogether we obtained 80 responses for each condition per participant.

\subsection{Results}

\subsubsection{Response times}

The latencies of correct responses were entered into a three-factor ANOVA for repeated measurements on all factors: Visual field (LVF or RVF), target level (global or local), and congruency (congruent, neutral, or incongruent).

The ANOVA revealed a significant main effect of torget level, $F(1,15)=7.42, p<.05$, indicating that responses to the local leve] were faster ( $513 \mathrm{~ms}$ ) than those to the global level ( $546 \mathrm{~ms}$ ). Also the main effect of congruency was reliable, $F(2,30)=4.43, p<.05$. The response times for congruent, neutral, and incongruent stimuli were: 521,530 , and $538 \mathrm{~ms}$, respectively. There was also a significant interaction between visual field and target level, $F(1,15)=6.39$, $p<.05$, in the expected direction, as can be seen in Fig. 2. For the local level the responses to LVF-stimuli were slower ( $515 \mathrm{~ms}$ ) than to RVF-stimuli (511 ms), whereas the opposite held for the global

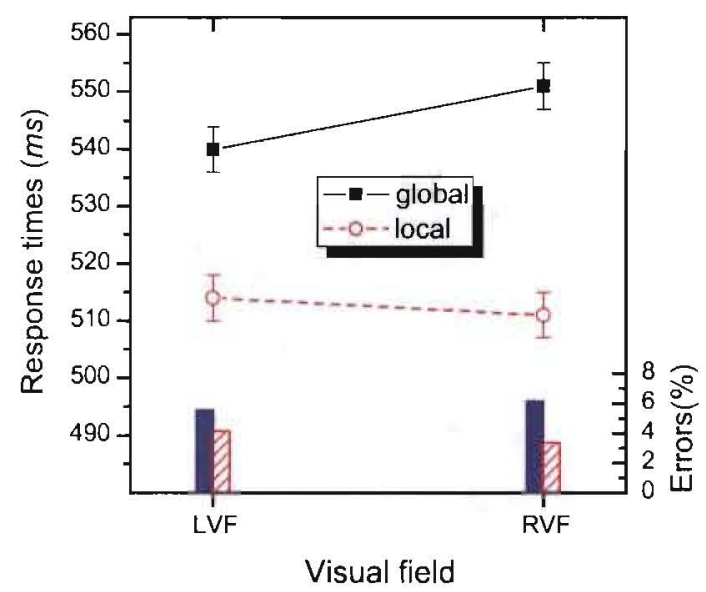

Fig. 2. Visual-field effects in Experiment 1. The error bars for the mean response times show the standard error of the mean for the level-specific comparisons. level ( $540 \mathrm{~ms}$ vs. $551 \mathrm{~ms}$ ). Planned comparisons revealed that the individual VF-effect was significant for the global level, $t(47)=2.45, p<.01$, but not for the local level, $t(47)=0.920$, $p=.181$.

\subsubsection{Error rates}

Errors occurred, on average, in $4.80 \%$ of the trials. They were subjected to an ANOVA of the same type as the response times. It turned out that the main effects of target level, $F(1,15)=13.6, p<.01$, and of congruency, $F(2,30)=34.1, p<.001$, were significant. Responses to the global level produced more errors than those to the local level ( $5.85 \%$ vs. $3.76 \%$ ). The error rates for congruent, neutral, and incongruent stimuli were: $2.42,3.62$, and $8.38 \%$, respectively. The twoway interaction between visual field and target level pointed in the expected direction, and was marginally significant, $F(1,15)=4.25$, $p=.057$ (see Fig. 2). Planned comparisons revealed that the VF-effect was significant for the local level, $t(47)=1.89, p<.05$, but not for the global level, $t(47)=1.24, p=.111$.

\subsection{Discussion}

The results show that VF-effects for global/local processing can also be obtained with naturalistic stimuli (see Fig. 2). All VF-effects point in the expected direction, and the effect for the global level was reliable in the response times, whereas that for the local level was reliable in the error rates. The different congruency conditions had no modulating effect. Thus, we can conclude that the VF-effects observed with hierarchal letters can also be observed with more naturalistic objects.

Although our VF-effects are similar to those observed with hierarchical letters, one might hesitate to acknowledge that they were due to a hierarchical global/local relation between animal shapes and pattern elements. With hierarchical letters, where the same content appears at both levels, it is evident that only the position in the hierarchical structure (global or local) can be responsible for the lateralization. With our naturalistic stimuli, though, the shape of the animals can occur only at the global level and the pattern elements only at the local level. As the shape of the animals is completely independent of the elements, both components might also have been coded independently (cf. Kimchi, 1992) and, therefore, their processing might also be lateralized independently of the hierarchical context. In this case, if the animals and pattern elements are presented in isolation, one would expect to find the same VF-effects. If, however, the hemispheric differences do indeed reflect processing asymmetries for the processing of global 
and local information of a hierarchical stimulus, then no VF-effects should occur for animals and element patterns presented in isolation. This question was addressed in the next experiment.

\section{Experiment 2}

In this experiment, we investigated whether the VF-effects observed in Experiment 1 do also occur when animals and pattern elements are displayed separately across trials. At least for the animals such a result would not be unreasonable, because they already constitute some kind of hierarchical object, even without a pattern. The situation is different for the patterns. At least with respect to global/local research there is some evidence that VF-effects do not occur for isolated local patterns (cf. Christman \& Weiner, 1997; Kimchi \& Merhav, 1991). In any case, the result of the present experiment should provide some information about the role of combining shapes and patterns for the VF-effects observed in Experiment 1.

As stimuli for the animal categorization task we simply used shaded dogs and cats without any pattern. But what should be used as stimuli for the element task? If we had presented a whole pattern, then this could have induced a virtual global contour that might have substituted the global animal shape. Thus, we might have observed the same VF-effect as in Experiment 1, but for a different reason. That is, the elements might now have been coded as local elements relative to a global virtual contour. However, this is not what we wanted to test. On the other hand, presenting a single element would have reduced the compatibility with Experiment 1 . Finally, as a compromise, we decided to use isolated pairs of lines and dots for the element categorization task (for examples see Fig. 3 ). The elements were presented in a brown color on a blank black screen.

Because animals and pattern elements were separated, it makes little sense to refer to them as "global level" and "local level", respectively. Therefore, we call the corresponding factors "animal task" and "element task". If the animals and pattern elements in Experiment 1 were hierarchically coded as the global and local level of a common object, respectively, and if this relation is important for the VF-effects to occur, then we should observe no VFeffects for the isolated components.

\subsection{Method}

\subsubsection{Subjects}

Sixteen persons ( 15 female; mean age 23.1 years) participated in the experiment. All were right-handed (by self report), and

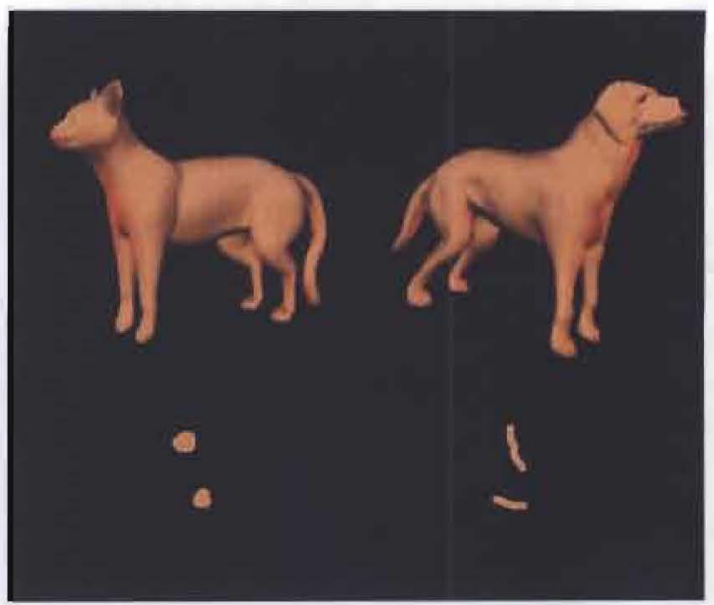

Fig. 3. Examples of the stimuli presented in Experiment 2. had normal or corrected-to-normal vision. None of the subjects had participated in Experiment 1.

\subsubsection{Stimuli and procedure}

The equipment and the procedure were the same as in the previous experiment. Also the stimuli were similar. Here, however, the patterns consisted of only two line or dot elements, respectively (for examples see Fig. 3). Their color was brown and they were presented on a black background. They were presented at an eccentricity that corresponded to that of the innermost pattern elements in the previous experiment.

\subsection{Results}

\subsubsection{Response times}

The latencies of correct responses were entered into a 2-factor ANOVA for repeated measurements on the factors visual field (LVF or RVF), and task (animal or element).

The analysis revealed a significant main effect of task, $F(1,15)=50.1, p<.001$, indicating that the pattern elements were categorized faster than the animals ( $434 \mathrm{~ms}$ vs. $496 \mathrm{~ms}$ ). This time, the interaction between visual field and task was far from significance, $F(1,15)=0.070, p=.795$. As can be seen in Fig. 4 , for the animal task there was even a small trend in the opposite direction.

\subsubsection{Error rates}

Errors occurred, on average, in $5.78 \%$ of the trials. They were subjected to an ANOVA of the same type as for the response times. It revealed a significant main effect of task, $F(1,15)=7.08, p<.05$. There were more егrors for the animal task than for the element task (6.72\% vs. $4.84 \%)$. The interaction between visual field and task was not significant, $F(1,15)=2.87, p=.11$.

\subsubsection{Comparison with Experiment 1}

An ANOVA including the data of Experiments 1 and of 2 revealed a significant three-way interaction in response times between level, VF, and experiment, $F(1,30)=4.71, p<.05$.

\subsection{Discussion}

In the present experiment, it was tested whether the VF-effects observed in Experiment 1 also occur when the animals and pattern elements are presented separately across trials. As the results clearly show, this was not the case (see Fig. 4). There was no signif-

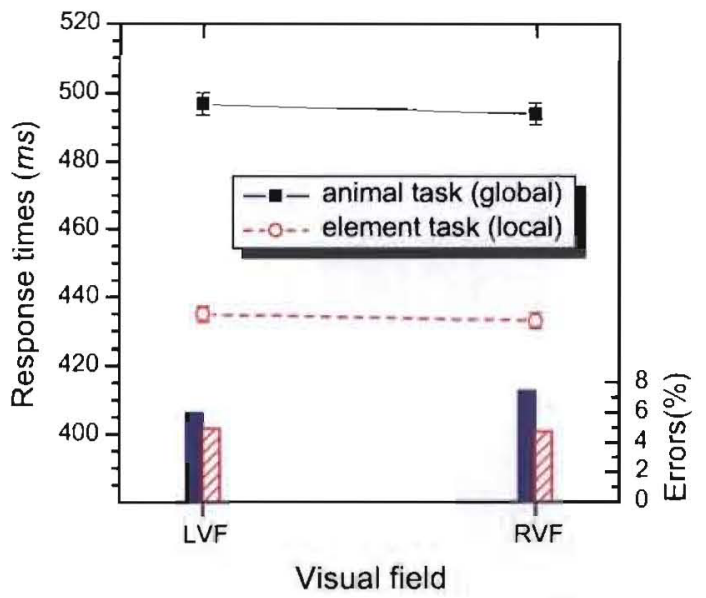

Fig. 4. The results of Experiment 2. The error bars for the mean response times show the standard error of the mean for the task-specific comparisons. 
icant interaction between visual field and task. Although Fig. 4 shows that there was a trend in the correct direction in the error rates, for the animal task this trend was not only unreliable, but was also counteracted by an opposite trend in the response times. Thus, our results indicate that it is essential for obtaining reliable VF-effects for global/local processing with our stimuli that the animal shapes and the pattern elements are mentally represented as the global and local levels of a common object, respectively.

The result that animals did not produce hemispheric asymmetries even without the pattern was not necessarily expected, because animal shapes are actually hierarchical objects per se as they could be conceptualized as being composed of constituent parts. The present results, however, confirm that animal shapes can indeed be perceived holistically without independent representations of their constituent parts (e.g Arguin \& Saumier, 2004; Behrmann et al., 2006; Humphreys et al., 1994; Johnston \& Hayes, 2000; Laeng et al., 1999; Large \& McMullen, 2006).

With respect to the local level it might be criticized that we reduced the number of pattern elements, and that this impedes the comparability with Experiment 1 . However, even if this would have been the case, such a critique does not hold for the animal shapes, because they were identical in the two experiments. If at all, then one would have expected an effect for these animal stimuli. However, also for the animal categorization task there was no VF-effect. Thus, taken together the data suggest that, if animals are shown with patterns on their body, the shape of the animal and the pattern elements are coded hierarchically. It seems that, only if the task-relevant components are part of a common global/local relation, they produce corresponding VFeffects.

\section{Experiment 3}

Our first experiments demonstrate that VF-effects can also be obtained with natural objects, provided that the task-relevant components are coded hierarchically. However, there is one difference between our results and those usually found in studies using hierarchical letters as stimuli. With hierarchical letters there is usually no local advantage. Rather, the levels are equal in dominance or there is even a global advantage, i.e. the responses to the global level are faster than those to the local level, and the interference from global to local is stronger than that from local to global (e.g. Hübner, 1997; Hübner \& Malinowski, 2002). In Experiment 1, however, although the interference did not differ between the levels, the categorization of the local pattern elements was faster than that of the global animal shapes. Thus, it remains open whether this local advantage was related in some way to the VF-effects observed in Experiment 1. Although there is no reason to assume that a local advantage is favorable for observing VFeffects with our more natural stimuli, for reasons of generality it would be desirable to see that the VF-effects do not depend on a specific dominance relation between the levels.

To see whether VF-effects also occur if the local level is less dominant, we had to create appropriate stimuli. The local advantage in Experiment 1 was presumably due to the fact that lines can easily be discriminated from dots. Therefore, relative to Experiment 1, we now exchanged the roles of dots and ovals: Dots were used as neutral elements and ovals as relevant elements. Consequently. the task for the local level was now to categorize the elements as lines or ovals. Because both element types are elongated, they should be more difficult to discriminate than dots and lines. Accordingly, we expected a global advantage, or, at least a reduced local advantage. If the dominance relation between the levels is irrelevant for hemispheric differences, then we should observe the same pattern of VF-effects as in Experiment 1.

\subsection{Method}

Twenty-four persons (20 female; mean age 23 years) participated in the experiment. All were right-handed (by self report), and had normal or corrected-to-normal vision. None of the participants had participated in any other experiment of this study.

The stimuli and the procedure were the same as in Experiment 1. The only difference was that lines and ovals were used as taskrelevant pattern elements, whereas dots served as neutral elements. The local task was modified accordingly.

\subsection{Results}

\subsubsection{Response times}

The latencies of correct responses were first entered into a three-factor ANOVA for repeated measurements on all factors: Visual field (LVF or RVF), target level (global or local), and congruency (congruent, neutral, or incongruent).

The analysis revealed a significant main effect of congruency. $F(2,46)=5.67, p<.01$, (congruent: $535 \mathrm{~ms}$; neutral: $545 \mathrm{~ms}$; incongruent: $548 \mathrm{~ms}$ ). However, the effect of congruency was further qualified by a two-way interaction between the factors level and congruency, $F(2,46)=11.3, p<.001$. It indicates that global-to-local interference (congruent: $529 \mathrm{~ms}$; neutral: $553 \mathrm{~ms}$; incongruent: $549 \mathrm{~ms}$ ) was much more pronounced than local-to-global-interference (congruent: $542 \mathrm{~ms}$; neutral: $538 \mathrm{~ms}$; incongruent $547 \mathrm{~ms}$ ). Most importantly, the interaction between visual field and target level was significant, $F(1,23)=9.64, p<.01$, For the local level the responses to LVF-stimuli were slower $(550 \mathrm{~ms})$ than to RVF-stimuli ( $537 \mathrm{~ms}$ ), whereas the opposite held for the global level ( $540 \mathrm{~ms}$ vs. $544 \mathrm{~ms}$ ). Planned comparisons revealed that the VF-effect was significant for the local level, $t(47)=3.31, p<.01$, but not for the global level, $t(47)=1.16, p=.128$ (see Fig. 5 ).

\subsubsection{Error rates}

Errors occurred, on average, in $3.53 \%$ of the trials. They were subjected to an ANOVA of the same type as the response times. The main effect of target level was significant, $F(1,23)=4.84$, $p<.05$. More errors occurred for the global level than for the local level ( $3.89 \%$ vs. $3.15 \%$ ). Also the congruency factor produced a reliable effect, $F(2,46)=32.5, p<.001$, (congruent: $2.33 \%$; neutral: 2.92\%; incongruent: $5.34 \%$ ). No other effects were significant. The interaction between visual field and target level was marginally significant, $F(1,23)=3.73, p<.066$. For the local level more errors occurred for LVF-stimuli $(3.45 \%)$ than for RVF-stimuli $(2.86 \%)$,

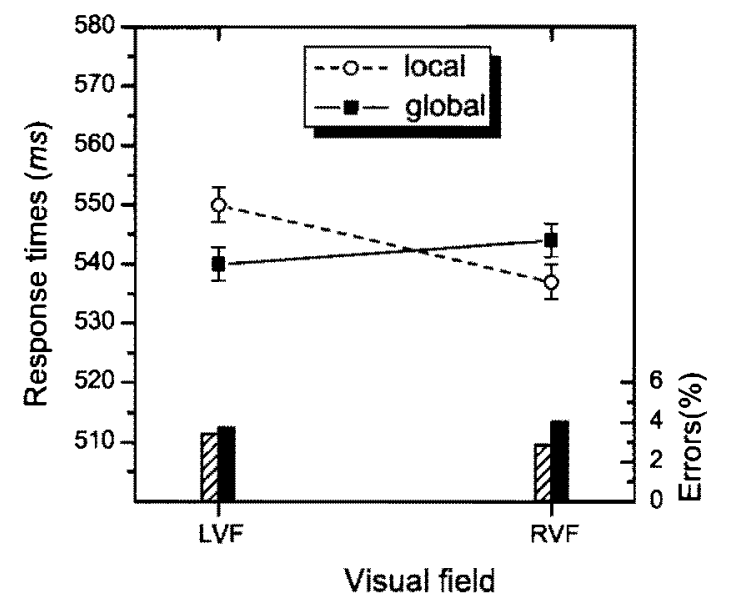

Fig. 5. The VF-effects in Experiment 3. The error bars for the mean response times show the standard error of the mean for the level-specific comparisons. 
whereas the opposite held for the global level ( $3.5 \%$ vs. $4.05 \%$ ). Planned comparisons revealed that the VF-effect was marginally significant for the local level, $t(47)=1.47, p=.077$, but unreliable for the global level, $t(47)=0.901, p=.188$.

\subsection{Comparison of Experiment 1 and Experiment 3}

In order to see whether the level dominance and the VF-effects differed berween Experiments 1 and 3, the data were analyzed together. The considered factors were experiment (1 or 3), visual field (LVF or RVF), and target level (global or local).

\subsubsection{Response times}

The analysis revealed a significant interaction between target level and experiment, $F(1,38)=6.78, p<.05$. It indicates that responses to the local level were faster in Experiment 1 than in Experiment $3(513 \mathrm{~ms}$ vs. $544 \mathrm{~ms}$ ), whereas the responses to the global level showed a trend in the opposite direction (546 ms vs. $542 \mathrm{~ms}$ ). The interaction between visual field and target level was highly significant, $F(1,38)=15.8, p<.001$, and did not significantly differ between the experiments, $F(1,38)=0.186, p=.668$. Planned comparisons revealed that the VF-effect was significant for the global level, $t(119)=2.78 . p<.01$, and for the local level, $t(119)=3.85$, $p<.01$.

\subsubsection{Error rates}

The analysis revealed a significant main effect of experiment, $F(1.38)=5.46, p<.05$. It indicates that more errors were made in Experiment 1 than in Experiment 3 . There was also a significant main effect of target level, $F(1,38)=17.8, p<.001$, which however, was qualified by a significant interaction between target level and experiment, $F(1,38)=4.75, p<.05$. It indicates that for the global level more errors were made in Experiment 1 than in Experiment 3 (5.84\% vs. $3.89 \%$ ), whereas the difference was less pronounced for the local level ( $3.76 \%$ vs. $3.15 \%)$. Most importantly, the interaction between visual field and target level was significant, $P(1,38)=7.98$, $p<.01$, and did not significantly differ between the experiments, $F(1.38)=0.434, p=.514$. Planned comparisons revealed that the VF-effect was significant for the local level, $t(119)=2.39, p<.01$, and marginally significant for the global level, $t(119)=1.54$, $p=.063$.

\subsection{Discussion}

As expected, using lines and ovals as relevant pattern elements for the local task, the local advantage vanished, compared to Experiment 1 , where lines and dots had been used. This confirms our hypothesis that lines and dots are easier to discriminate than lines and ovals. Although the responses to the global level were only slightly faster than those to the local level ( $562 \mathrm{~ms}$ vs. $567 \mathrm{~ms}$ ), with respect to congruency, there was now a global advantage, i.e. the interferences from global to local was significantly stronger than that from local to global. Most importantly, despite the global advantage, the VF-effects showed a similar pattern as in Experiment 1 (see Fig. 5). This indicates that the VF-effects for our naturalistic stimuli do no depend on a specific level dominance.

\section{General discussion}

The present study was concerned with functional hemispheric differences with respect to global/local processing. Usually, corresponding VF-effects are investigated with hierarchical letters (for overviews see Hübner \& Volberg, 2005; Van Kleeck, 1989; Yovel et al., 2001). Their predominant use is mainly due to the fact that these stimuli are not only relatively easy to construct, but that the content of the levels can also be exchanged and varied independently (Pomerantz, 1981). Although these properties have many advantages from an experimental point of view, they are also rather specific, which might severely restrict the generality of the abtained results. Thus, the aim of the present study was to examine whether similar level-specific VF-effects can also be obtained with more naturalistic stimuli.

A great challenge was to find appropriate stimuli. Results on object recognition suggest that the global level of an object corresponds to its overall shape. More difficult, though, was to determine the component that represents the local level. The figural parts of a natural object are inappropriate for our objective, because they usually provide cues for the object category. Moreover, because the parts constitute the overall shape, they cannot be varied independently. In view of these constraints, we constructed images of animals with a pattern on their body surface. The task for the global level was to categorize the shown animal, while the task for the local level was to categorize the pattern elements.

In our first experiment, we presented our naturalistic stimuli and examined whether they produced the expected VF-effects with respect to global/local processing. Our results show that this was indeed the case, Reliable VF-effects occurred for both levels. However, despite this success it remained unclear whether our stimuli addressed the same processes usually involved in the processing of hierarchical letters. Obviously, our animal stimuli can still be considered as hierarchical objects even if the pattern is removed. Thus, the question was whether VF-effects would also occur if animal shapes and patterns were presented separately. This possibility was examined in Experiment 2. It turned out that the isolated shapes and patterns did not produce VF-effects. ${ }^{2}$ This implies that the animal shapes and pattern elements in Experiment 1 had indeed been coded as levels of a common object, and that such a coding is necessary for VF-effects to show up.

Thus, the results of our first two experiments suggest that the VF-effects observed with our natural stimuli are based on the same hemispheric differences as the VF-effects observed with hierarchical letters. However, compared to other VF-studies, the results of Experiment 1 are different with respect to the relative dominance of the levels. With hierarchical letters as stimuli, the responses to the global level are often faster than those to the local level, or at least equally fast (e.g. Yovel et al., 2001), and the interference from global to local is stronger than the interference from local to global. In Experiment 1, though, the interference between the levels was equally strong, and the responses to the local level were even faster than the responses to the global level. To test whether this local advantage was in any way responsible for the observed VF-effects, a further experiment was conducted in which the same animals were displayed as in the previous experiments. This time, however. lines and ovals had to be categorized as local task, instead of lines and dots. It was expected that the discrimination between lines and ovals would be more difficult than the discrimination berween dots and lines, and that this would slow down the responses to the local level. This was indeed the case. There was no local advantage in Experiment 3. Rather, although there was no response time difference between the levels, the interference form global to local was this time stronger than the interference from local to global. Nevertheless, a reliable interaction between level and VF occurred,

\footnotetext{
${ }^{2}$ One of the reviewers raised the question of whether the small proportion of male subjects in this experiment could be responsible for the null effect, because it has been hypothesized that men are more lateralized than women (Harris, 1978). However such an account is rather unlikely. First of all, several studies have shown that, if sex differences exist at all, they are extremely small (cf. Boles, 2005; Hiscock, Israelian, Inch, Jacek, \& Hiscock-Kali, 1995). Furthermore, in our other experiments, where VF-efects were found, males were also in the minority.
} 
which did not differ from that in Experiment 1 This demonstrates that the specific dominance relation between the levels is unimportant for the VF-effects.

Altogether, the results of the present study demonstrate that the VF-effects observed for the global/local processing of hierarchical letters can also be obtained for naturalistic stimuli if appropriate stimuli and tasks are employed (for a related result in the context of a categorization task see Studer \& Hübner, in press). Although the level-specific VF-effects were relatively small and varied to some extent in size across Experiments 1 and 3, on average, they were reliable. This is good news for all researchers concerned with hemispheric asymmetries for global/local processing. They can continue their research with abstract hierarchical letters without worrying that their results cannot be generalized to naturalistic objects.

However, we also have to concede that the generalization in the present study is restricted to living objects. Thus, an interesting question is: Would we expect the same outcome for non-living objects? If we consider the members of basic categories, then it turns out that the global shapes of non-living objects within a basic category usually show a greater variability than those of living things, because non-living things are more defined by their function than by their visual appearance (e.g. Thomas \& Forde, 2006). For instance, the shapes of different lamps vary to a high degree, whereas all kinds of birds look rather similar. Thus, it is plausible to assume that living objects and non-living objects are categorized by attending to their global shape or by attending to their local details, respectively. This hypothesis is supported by results showing that the right hemisphere exhibits an advantage for the categorization of living objects (e.g. Laeng et al., 1999), whereas, for the identification of non-living objects, the left hemisphere shows an advantage (e.g. Lag et al., 2006). Thus, these results for the local level of non-living objects are in line with the present data. In a global/local task with non-living objects, however, it is clear that subjects would also have to pay attention to the global level of the non-living objects on corresponding trials, which should involve the right hemisphere. Thus, it is likely that in a global/local task an interaction between visual field and level would occur similarly to the one in the present study for living objects. Whether this is indeed the case, however, has to be shown in further studies.

A further aspect of that bears mention is that the naturalistic stimuli applied in the present study seem to have a favorable property compared to hierarchical letters. With hierarchical letters, VFeffects are often restricted to incongruent stimuli (e.g. Hübner \& Malinowski, 2002; Van Kleeck, 1989), at least in focused attention paradigms (cf. Hübner, Volberg, \& Studer, 2007). In contrast, the VF-effects in the present study did not depend on a specific congruency condition. Thus, the application of our stimuli seems to generally increase the probability of observing VF-effects for global/local processing.

The observation that, if hierarchical letters are used as stimuli, VF-effects do not occur in congruent conditions (i.e. if the global and local level activate the same response), can be explained by the integration theory of global/local processing (Hübner \& Volberg. 2005). This theory assumes that the letters of a hierarchical letter can already be identified at an early stage of processing. where the hemispheres do not differ. It is proposed that the hemispheres only differ at a later stage, where the object features are integrated into a more complete object representation. Because congruent stimuli activate the same response, a response can already be selected by the information available at the early stage, which explains why there are no VF-effects for these stimuli. Only when the letters activate competing responses, which is the case for incongruent stimuli, information from the late stage is needed to select the correct response, and this stage is where the VF-effects originate (for details see Hübner \& Volberg, 2005). Thus, according to the integration theory, the present results indicate that response selection for our natural objects is always based on a relatively complete object representation. For instance, it is possible that natural objects automatically lead to an initial high-level percept and that further processing is then required to extract information for specific tasks (cf. Ahissar \& Hochstein, 2004). Subsequent research has to show whether this is indeed the case.

In any case, as far as we know, the present study is the first with naturalistic stimuli that reports the proposed VF-effects for global/ local processing. Thus, our results show that the functional hemispheric differences for global/local processing observed for hierarchical letters can also be generalized to naturalistic stimuli.

\section{Acknowledgment}

This research was supported by a grant from the Deutsche Forschungsgemeinschaft (DFC) to the first author (Hu 432/11).

\section{References}

Ahissar, M., \& Hochstein, S. (2004). The reverse hierarchy theory of visual perceptual learning. Trends in Cognitive Sciences, $8,457-464$

Arguin, M. Saumier, D. (2004). Independent processing of parts and of their spatial organization in complex visual objects. Psychological Science, 15. $629-633$.

Behrmann, M. Feterson, M. A., Moscovitch, M., \& Suzuki, S. (2006) Independent representation of parts and the relations between them: Evidence from integrative agnosia, Joumal of Experimental Psychology: Human Perception and Performance, 32, 1169-1184.

Boles. D. B. (2005). A large-sample study of sex differences in functional cerebral lateralization. Joumal of Clinical and Experimental Neuropsychology, 27, 759-768.

Bradshaw, J. L., \& Netteton, N.C. (1981), The nature of hemispheric specialization in man. The Behavioral and Brain Sciences, 4, 51-91.

Christman, S. $D_{x,}$ \&einer, $R, H$. (1997). Hernispheric processing of form versus texture at the local level of hierarchical patterns. Acta Psychologica, 96, 193-206.

Delis, D. C., Robertson, L. C., \& Efron, R. (1986). Hemispheric specialization of memory for visual hierarchical stimuli. Neuropsychologia, 24, 205-214.

Fink, G. R., Halligan, P. W., Marshall, J. C., Frith, C. D., Frackowiak, R. S. J, \& Dolan, R. J (1996). Where in the brain does visual attention select the forest and the trees? Nature, 382, 626-628.

Fink, G. R, Halligan P W Marshall, I C Frith, C D. Frackowiak, R S J \& Dolan, R I (1997). Neural mechanisms involved in the processing of global and local aspects of hierarchically organized visual stimuli. Brain. $120.1779-1791$.

Han, S., Fan, S, Chen, L, \& Zhuo, Y. (1999). Modulation of brain activities by hierarchical processing: A high-density ERP study. Brain Topography, 11 $171-183$.

Harris, L. 1. (1978). Sex differences in spatial ability: Possible environmental, genetic, and neurological factors. In $\mathrm{M}$. Kinsboume (Ed.). Asymmetrical function of the brain (pp. 405-522), Cambridge, UK: Cambridge University Press.

Heinze, H. J. Hinrichs, H., Scholz, M. Burchert, W., \& Mangun, G. R. (1998). Neural mechanisms of global and local processing: A combined PET and ERP study. Journal of Cognitive Neuroscience, 10, 485-498.

Heinze, H. J. \& Mïnte, T.F. (1993). Electrophysiological correlates of hierarchical stimulus processing: Dissociation between onset and later stages of global and locat target processing. Neuropsychologia, 31,841-852.

Hiscock, M., Israelian. M. Inch, R. Jacek, C., Hiscock-Kalil. C. (1995). Is there a sex difference in human laterality? II. An exhaustive survey of visual laterality studies from six neuropsychology journals. Journal of Clinical and Experimental Neuropsychology, 17, 590m610.

Hübner, R. (1997). The effect of spatial frequency on global precedence and hemispheric differences. Perception 8 Psychophysics, 59, 187-201.

Hübner, R. Malinowski, P. (2002). The effect of response competition on functional hemispheric asymmetries for global/local processing. Perception $E$ Pychophysics, 64, 1290-1300.

Hübner, R., \& Volberg, G. (2005). The integration of object levels and their content: $A$ theory of global/local processing and related hemispheric differences. Joumal of Experimental Psychology: Human Perception and Performance, 31, 520-541.

Hubner, R. Volberg, G. \& studer, T. (2007). Hemispheric differences for global/local processing in divided attention tasks: Further evidence for the integration theory. Perception E Psychophysics, 413-421.

Humphreys, G. W. (1998). Neural representation of objects in space: A dual coding account Philosophical Transactions of the Royal Society. Series B, Biological Sciences, 353, 1341-1351.

Humphreys, $G$. W., Riddoch, M. . . Donnelly, N., Freeman, T., Boucart, M. \& Müller, H. M. (1994), Intermediate visual processing and visual agnosia. In M. J. Farah \& $G$. Ratciff (Eds.). The neuropsychology of high-level vision (pp. 63-101). Hillsdale, N]: Erlbaum.

Johnston, M. B., \& Hayes, A. (2000). An experimental comparison of viewpointspecific and viewpoint-independent models of object representation. Quarterly Journal of Experimental Psychology, 53(A), 792-824 
Kimchi, R. (1992). Primacy of wholistic processing and global/local paradigm: A critical review. Aychological Bulletin, 112, 24-38.

Kimchi, R. Merhav, 1.(1991). Hemispheric processing of global form, local form, and texture. Acta Psychologica, 76, 133-147.

Laeng. $B_{*}$ Shah, J., Kosslyn, S. (1999). Identifying objects in conventional and contorted poses: Contributions of hemisphere-specific mechanisms. Cognition $70,53-85$.

Lag, T. Hveem, K, Rutud, K. P. E, Laeng, B. (2006). The visual basis of category effects in object identification: Evidence from the visual hemifield paradigm. Brain and Cognition, 60, 1-10.

Large, M.-E., \& McMullen, P. A. (2006). Hierarchical attention in discriminating objects at different levels of specificity. Perception \& Psychophysics, 68, $845-860$.

List, A. \& Justus, T. (2007). Auditory priming of frequency and temporal information: Effects of lateralised presentation. Laterality, 12,507-535.

Lux, S., Marshall, A., Weiss, P. H., Pietrzyk, U., Shah, N. J., Zilles, K. et al. (2004). A functional magnetic resonance imaging study of local/global processing with stimulus presentation in the peripheral visual hemifields. Neuroscience, 124 $113-120$.

Lux, S., Thimm, M. Marshall, J. C, \& Fink, G. R. (2006). Directed and divided attention during hierarchical processing in patients with visuo-spatial neglect and matched healthy volunteers. Neuropsychologia, 44, $436-444$.

Malinowski, P., Hubner, R., Keil, A, \& Gruber, T. (2002). The influence of response competition on cerebral asymmetries for processing hierarchical stimuli revealed by ERP recordings. Experimental Brain Research. 144, 136-139.
Martin, M. (1979). Hemispheric specialization for global and local processing. Neuropsychologia, $17,33-40$.

Navon, D. (1977). Forest before the trees: The precedence of global features in visual perception. Cognitive Pychology, 9, 353-393.

Pomerantz, ]. R. (1981). Perceptual organization in information processing. in $\mathrm{M}$. Kubovy ]. R. Pomerantz (Eds.), Perceptual organization. Hillsdale, N.:: Erlbaum

Robertson, L. C., Lamb, M. R. (1991). Neuropsychological contributions to theories of part/whole organization. Cognitive Psychology, 23, 299-330.

Studer. T. \& Hübner. R. (in press). The direction of hemispheric asymmetries for object categorization at different levels of abstraction depends on the task. Brain and Cognition, doi: $10.1016 / \mathrm{j}$.bandc,2008.01 .003.

Thomas, R. \& Forde, E. (2006). The role of local and global processing in the recognition of living and nonliving things. Neuropsychologia. 44. 982-986.

Tyler, L. K.. Stamatakis, E. A., Bright, P., Acres, K., Abdallah, S., Rodd, J. M., et al (2004). Processing objects at different levels of specificity. Joumal of Cognitive Neuroscience, 16, 351-362.

Van kleeck, M. H. (1989). Hemispheric differences in global versus local processing of hierarchical visual stimuli by normal subjects: New data and a meta-analysis of previous studies. Neuropsychologia, $27,1165-1178$.

Volberg. G. Hubner, R. (2004). On the role of response conflicts and stimulus position for hemispheric differences in global/local processing: An ERP study. Neuropsychologia, 42, 1805-1813.

Yovel, $G$., Yovel, I, \& Levy. I. (2001). Hemispheric asymmetries for global and local visual perception: Effects of stimulus and task factors. Joumal of Experimental Pychology: Human Perception and Perfonnance, 27, 1369-1385. 